\title{
Impact of Mobility Models on DLA (Drop Largest) Optimized DTN Epidemic routing protocol
}

\author{
Sulma Rashid, Qaisar Ayub, M. Soperi Mohd Zahid, A.Hanan. Abdullah \\ Faculty of Computer Science \& Information System, \\ Department of Computer System \& Communication \\ Universiti Teknologi Malaysia (UTM) Skudai - Johor, 81310, Malaysia.
}

\begin{abstract}
DTN mobile nodes depend on their mobility to carry the message to destination. Therefore it is important to understand the effect of buffer management policies on the performance of DTN routing protocols under different mobility models.

In our previous work of DLA we examine that epidemic router was not showing good delivery probability in case of SPMBM. This paper is the performance of DLA (drop largest) and DOA (Drop oldest) buffer management policy with impact of varying mobility models under epidemic routing protocol. We show that how combination of mobility models and queuing mechanism can optimize the performance of epidemic routing protocol in term of delivery probability, message dropped, buffer time average, overhead ratio and hop count averages.
\end{abstract}

\section{General Terms}

Routing, Mobility Models, Epidemic Router.

\section{Keywords}

DTN (Delay Tolerance Network), Queuing Policies, store-carryforward, Drop Largest, message drop policies, mobility models

\section{INTRODUCTION}

The traditional network architecture TCP/IP ,mobile ad hoc proactive, reactive required end-to-end path and can not be implemented in advanced wireless applications e.g. Military networks, vehicular ad hoc network, wild life tracking sensor networks.

In order to delivery the message in such environment researchers have proposed Delay Tolerance Network DTN [1], [2], [7] and [8] where the network infrastructure is composed of sparse nodes, further each node in the network stores a packet that has been forwarded to it by other nodes, carries the packet while it moves around, and forwards or duplicates it to other nodes (or the destination node) when they come surrounded by transmission range.

DTN routing with single-copy [21] approach reduce the buffer usage but suffers from long delays and low delivery ratio.

Multi-copy [3] and [4] schemes results in low delivery delay and gain high message delivery. In such schemes buffer quickly gets congested, so a good buffer management policy is required to overcome this congestion [17], [18],[15] and [22].

Further while moving according to mobility model the node continue to replicate the message on available links. The mobility of the nodes affects the number of average connected paths, which in sequence influence the performance of the routing algorithm Therefore combination of node mobility models with buffer management policy can optimize the results in our concern domain areas of disruption environment [19] and [20].

In our previous work of DLA[15] we inspect that epidemic router is not showing good delivery probability in case of SPMBM so in this work we explore epidemic routing with other mobility models . And we evaluate buffer management policy (DLA), Drop Oldest (DOA) with various mobility models under highly congested network. We have performed through the simulations that Epidemic routing protocol under five mobility models RWP, RW, SPMBM, MBM, and Manhattan.

The rest of paper is organized follows Section 2 discuss existing buffer management policies. Section 3 summarizes performance metrics. Section 4 describes evaluation our buffer management policy under routing protocols. Section 5 is about mobility models, Section 6 gives proposed algorithm (DLA). Section 7-8 is for simulation results and conclusion.

\section{EXISTING BUFFER MANAGEMENT POLICES}

\subsection{Drop Random (DR)}

The choice of message to be dropped is in arbitrary order.

\subsection{Drop-Least-Recently-Received (DLR)}

It drop the message that have been in the node longest.[16]

\subsection{Drop-Oldest (DOA)}

The message with the shorted remaining life time (TTL) in network is dropped. The idea of dropping such packet is that if packet TTL is small, it is in the network for long time and thus has high probability to be already delivered.

\subsection{Drop Tail}

It drops the newly arrive message.

\subsection{Drop Front}

The message that arrives first in the buffer is dropped first.

\subsection{N-Drop}

In N-Dropt [16], the message that does $\mathrm{N}$ number of forwarding will be selected to drop. 


\subsection{Drop Largest (DLA)}

In Drop Largest (DLA), large size message will be selected in order to drop. [15]

\subsection{MOFO- Evict most forwarded first}

The message that has been forwarded to maximum number of times will be dropped first. [14]

\subsection{MOPR - Evict most favorably forwarded first}

Each message in node is related with a forwarding predictability FP, initially assigned to 0 . When the message is forwarded the FP value is modified and the message with maximum FP value will be dropped first. [14]

\subsection{SHLI - Evict shortest life time first} The message contain smallest TTL will be selected to drop. [14]

\subsection{GBD (Global Knowledge based Drop)}

GBD based on global knowledge about the network state. As global Knowledge is required, GBD is difficult to be implemented, thus, it will serve as a point of reference. [13]

\subsection{HBD}

A deployable variant of GBD that uses the new utilities based on estimates of $m$ and $n$. [13]

\subsection{FBD (Flood Based Drop)}

FBD accounts only for the global information collected using simple message flooding, that is, without considering past history or other messages. [13]

\subsection{T-DROP}

Its will drop the message which lies in the threshold size range of buffer. [17]

\subsection{E-DROP}

Its will drop the message of equal size from the node buffer when the node congested [18].

\section{PERFORMANCE METRICS}

\subsection{Delivery Probability}

It is a function of measuring messages that are correctly received by the final destination within a given time period.

\subsection{Buffer Time Average}

The buffer time average is the function of measuring the average time spends by all messages in node buffer function of measuring the average time spends by all messages in node buffer.

\subsection{Message Drop}

It is a function to count number of messages dropped during transmission from the buffer.

\subsection{Overhead Ratio}

It is negation of number of message relayed to message delivered.

\subsection{Hop count Average}

The function of measuring the number of nodes a message accepted to reach the destination.

\section{PROTOCOL UNDER OBSERVATION}

\subsection{Epidemic Routing}

Epidemic Routing [6] spread application messages to nodes, called carriers, within connected areas of ad hoc networks. In Epidemic Routing when carriers coming into contact with another connected portion of the network through node mobility it spread the message to nodes. Through such iterative transmission of data, messages have a high probability of reaching their destination.

\section{MOBILITY MODEL UNDER OBSERVATION}

Various mobility models can be categorized according to their spatial and temporal dependencies.

Spatial dependency: It is a measure of how two nodes are dependent in their motion. If two nodes are moving in same direction then they have high spatial dependency.

Temporal dependency: It is a measure of how current velocity (magnitude and direction) are related to previous velocity.

Nodes having same velocity have high temporal dependency.

\subsection{Random Waypoint Model (RWP)}

The random way point [11] proposed by Johnson and lee works by moving the mobile nodes randomly according to direction speed at regular time intervals.

In this model a mobile node stays at location for certain period of time, once the time expired, the node moves to the new destination by choosing the random speed from [0MAXSPEED]. Hence node continues to move till the end of simulation along with crisscross path.

\subsection{Random Walk (RW)}

The random walk model [11] describes the individual's movement relative to cells. In random walk model a node moves from current location to a new location for a time interval $t$ by randomly choosing a direction and speed from the pre defined ranges. At the end of each time interval a new direction and speed are calculated. 
Table 01 Simulation Setup

\begin{tabular}{|c|c|c|c|c|c|}
\hline Parameters & 1 & 2 & 3 & 4 & 5 \\
\hline Number of Nodes & 126 & 1000 & 126 & 126 & 500 \\
\hline Movement model 1 & RWP & RW & SPMBM & MBM & Manhattan \\
\hline Movement model 2 & RMBM & MBM & RMBM & RMBM & RMBM \\
\hline Number of groups Model $1+2$ & $03+03$ & 01 & $03+03$ & $03+03$ & 03 \\
\hline Buffer size of Model 1+2 & $5 \mathrm{MB}+50 \mathrm{MB}$ & $5 \mathrm{MB}$ & $5 \mathrm{MB}+50 \mathrm{MB}$ & $5 \mathrm{MB}+50 \mathrm{MB}$ & $100 \mathrm{M}$ \\
\hline Transmission range & $10 \mathrm{M}$ & $10 \mathrm{M}$ & $10 \mathrm{M}$ & $10 \mathrm{M}$ & $10 \mathrm{M}$ \\
\hline Transmission speed & $250 \mathrm{~K}$ & $250 \mathrm{~K}$ & $250 \mathrm{~K}$ & $250 \mathrm{~K}$ & $100 \mathrm{k}$ \\
\hline Message creation interval & $25-35$ & $25-35$ & $25-35$ & $25-35$ & $25-35$ \\
\hline Message Range Random & $200 \mathrm{k}-2 \mathrm{MB}$ & $200 \mathrm{k}-2 \mathrm{MB}$ & $200 \mathrm{k}-2 \mathrm{MB}$ & $200 \mathrm{k}-2 \mathrm{MB}$ & $500 \mathrm{k}, 1 \mathrm{M}$ \\
\hline Simulations times varies & $200000 s$ & $200000 s$ & $200000 \mathrm{~s}$ & $200000 s$ & $200000 s$ \\
\hline Area of Simulation & $4500 \mathrm{~m} \times 3400 \mathrm{~m}$ & $4500 \mathrm{~m} \times 3400 \mathrm{~m}$ & $4500 \mathrm{~m} \times 3400 \mathrm{~m}$ & $4500 \mathrm{~m} \times 3400 \mathrm{~m}$ & $4500 \mathrm{~m} \times 3400 \mathrm{~m}$ \\
\hline TTL & Infinite & Infinite & Infinite & Infinite & $300 \mathrm{mins}$ \\
\hline
\end{tabular}

\subsection{Shortest Path map mobility (SPMBM)}

The Shortest path map based mobility model [8], SPMBM, is the extension of map based movement which uses the DijKstra's shortest path algorithm to calculate the shortest path from current location to randomly selected destination.

In SPMBM when a node reaches its destination it waits for the time interval $t$ and then directed to randomly selected node by using shortest path.

\subsection{Map mobility Models (MBM)}

In the simple random map based models [8], MBM, nodes move to randomly determined positions on the map but follow the roads to define by the map data.

\subsection{Route map mobility Models (RMBM)}

The route map mobility models are also extension of MBM but it's used for the vehicle mobility in groups. [8]

\subsection{Manhattan mobility model}

Manhattan model is used to simulate the movement pattern of mobile nodes on streets defined by maps [9] and can be applied to replicate movement in an urban area where portable devices are supported with stabilized computing service.

The map of Manhattan mobility model formulates a grid like structure of horizontal and vertical lines. This distribution is called streets and mobile node is allowed to move along the grid in horizontal or vertical. The mobile node is allowed to change its direction at a predefined probability i.e. on the same street is 0.5 , turning left is 0.25 , turning right is 0.25 .

\section{SIMULATION AND RESULTS}

In the following section we observe the performance of different mobility models with epidemic router with exiting (DOA), and Drop Largest (DLA). All the experiments were performed using ONE Simulator. The ONE [5] Simulator is a discrete event simulator written in Java. The main objective of simulator is to

Implement DTN (store-carry-forward) of message for long time, where the probability of disconnections and failures is high.

First we consider delivery probability of epidemic routing protocol with drop policies DOA, DLA with five mobility models as shown in figure 1 . We can see that DLA have varying impact on message delivery Probability, but higher delivery in case of RWP, Manhattan and RW. 


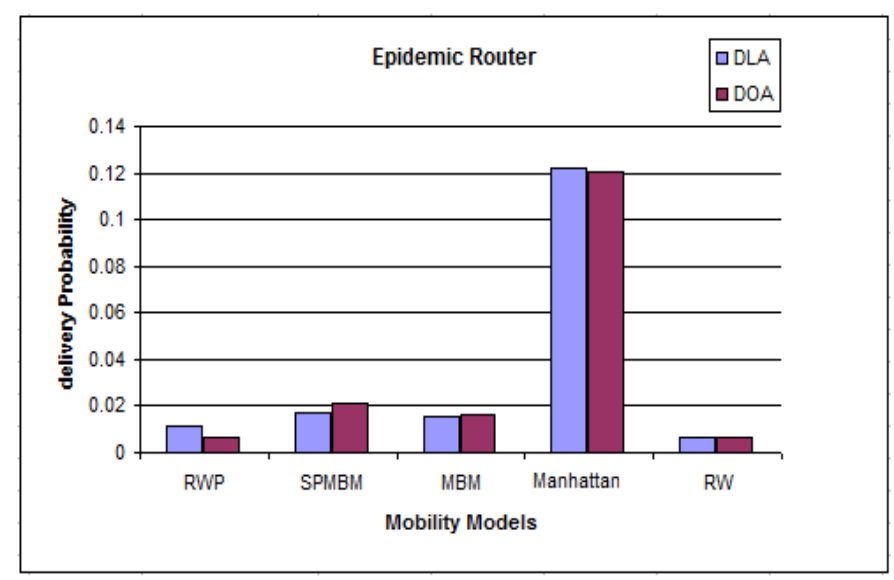

Figure 1 Delivery Probability

While in SPMBM and MBM DLA have less delivery probability than DOA. However in all the configuration of mobility models, message delivery of DLA is improved then DOA only affected in SPMBM and MBM but improves other metrics discussed later and is trade off.

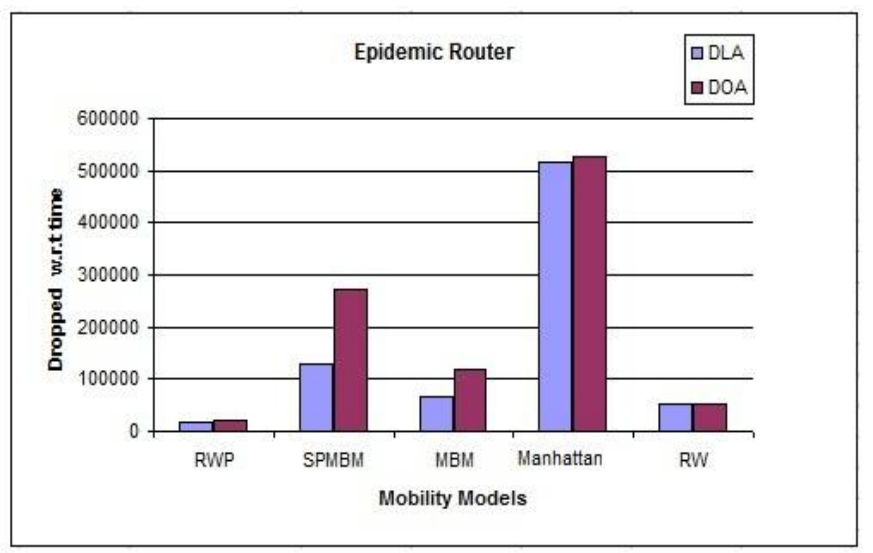

Figure 2 Message Dropped w.r.t Mobility Models

Figure 2 plot the impact of message dropped with DLA and DOA. In all Mobility models configurations DLA reduce the message drops to a significant quantity. We can observe the strength of DLA, which drop large size message and free more buffer space as described in algorithm [15] .In Epidemic routing the message delivery was affected in case of SPMBM and MBM due to the flooding nature of epidemic but message drop is fall down then existing DOA, DLA have optimized the message drop metric.

Figure 3 represents the influence of DLA and DOA with respect to overhead ratio with five mobility models. We can see clearly that overhead ratio with DLA is decrease in all mobility models with Epidemic router just equal in case of RW. Hence mobility models with DLA overhead is reduced to considerable extent.

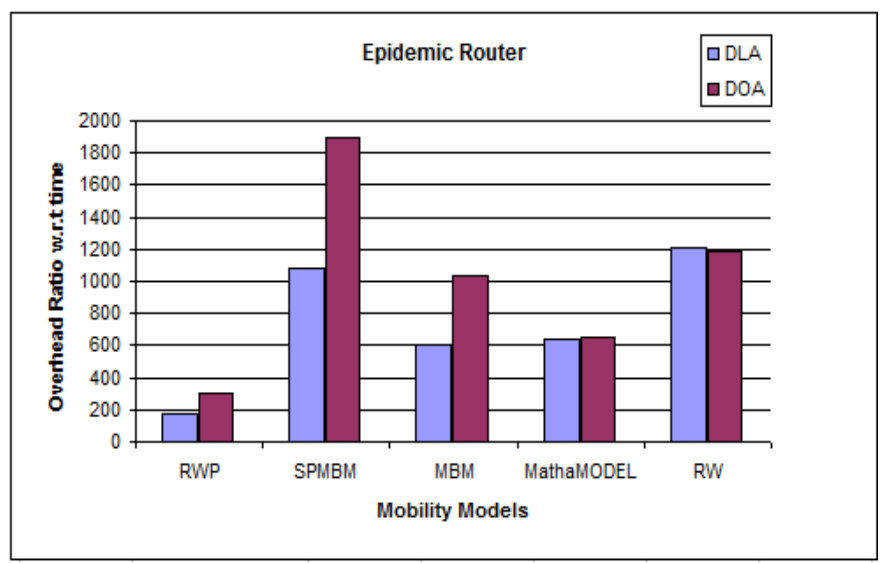

Figure 3 Overhead ratio w.r.t Mobility Models

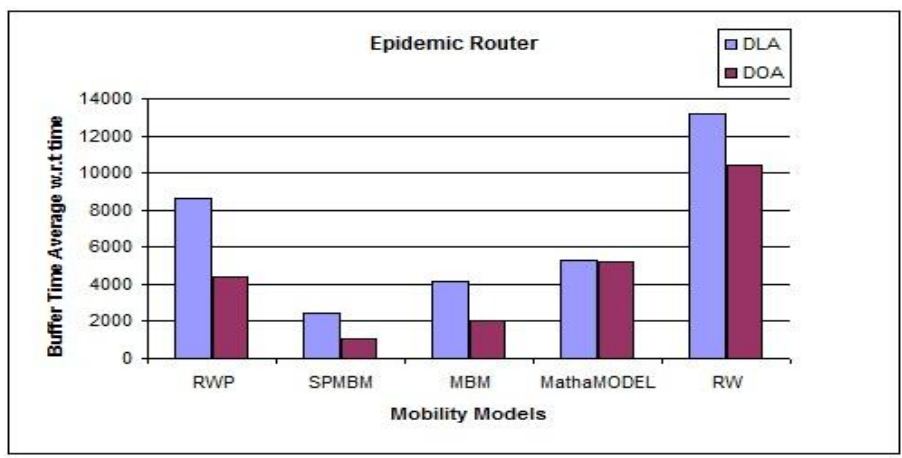

Figure 4 Buffer Time Average w.r.t Mobility Models

Figure 4 observes buffer time average with DLA and DOA under five mobility models. It can be clearly seen that DLA has high value of buffer time average with all mobility models. Higher value of buffer time indicates messages have high opportunity to stay in the buffer.

Figure 5 observes HOP Count Average with DLA and DOA. It can be clearly seen that DLA has low value of HOP Count Average with all mobility models. Its means the delivery probability is increase in DLA with less hop count average and message reached their destination earlier as compared to DOA and buffer was managed in more effective manner and more messages are accommodate and chance of delivery increases.

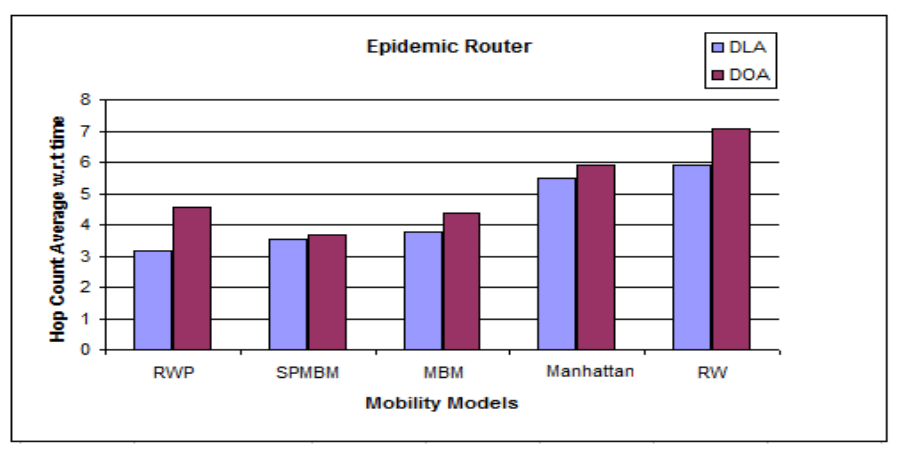

Figure 5 Hop Count average w.r.t Mobility Models 


\section{CONCLUSION \& FUTURE WORK}

In this paper we saw the impact of an efficient buffer management policy DLA which drop large size messages when the node buffer is congested with five mobility models RWP, RW, SPMBM, and MBM.

Its also optimize the router performance metrics like message delivery, message drop, overhead ratio and buffer time average compared to DOA. Future work is to investigate the DLA (Drop Largest) technique with all existing buffer management policies.

\section{REFERENCES}

[1] S. Jain, K. Fall, and R. Patra, "Routing in a delay tolerant network," in Proceedings of ACM SIGCOMM, pp.145158, 2004.

[2] T. Small and Z. J. Haas." Resource and performance trade offs in delay-tolerant wireless networks," in SIGCOMM Workshop on Delay Tolerant Networking (WDTN), pp.260-267, 2005.

[3] T. Spyropoulos, K. Psounis, and C. S. Ranghavendra "Spray and wait: an efficient routing scheme for intermittently connected mobile networks," in Proceedings of the ACM SIGCOMM workshop on Delay-tolerant networking.pp. 252-259,2005.

[4] T. Spyropoulos, K. Psounis, and C. Raghavendra. "Efficient Routing in Intermittently Connected Mobile Networks: The Multi-copy Case,'IEEE/ACM Transactions on Networking (TON), vol. 16, and pp. 77 - 90, Feb. 2008 .

[5] Homepage of Opportunistic Network Environment (ONE).http://www.netlab.tkk._/\%7Ejo/dtn/\#one Version 1, Accessed July 2010.

[6] A. Vahdat and D. Becker, "Epidemic routing for partially connected ad hoc networks," Duke University, Tech. Rep. CS-200006, Apr. 2000.

[7] K. Scott and S. Burleigh, "Bundle protocol specification." RFC 5050, November 2007.

[8] KERÄNEN, A., AND OTT, J. Increasing Reality for DTN Protocol Simulations. Tech. rep., Helsinki University of Technology, Networking Laboratory, July 2007.

[9] F. Bai, N. Sadagopan and A. Helmy, "IMPORTANT: A Framework to Systematically Analyze the Impact of Mobility on Performance of Routing Protocols for Ad Hoc Networks," Proceedings of the

[10] IEEE International Conference on Computer Communications, pp. 825-835, March-April 2003.
[11] Camp, T., J. Boleng, and V. Davies, A survey of mobility models for ad hoc network research. Wireless communications and mobile computing, 2002. 2(5): p. 483502 .

[12] Lindgren, A. Doria, and O. Schelen, "Probabilistic routing in intermittently connected networks," SIGMOBILE Mobile Computing and Communication Review, vol. 7, no. 3, 2003.

[13] Krifa, C. Barakat, and T. Spyropoulos.” Optimal buffer management policies for delay tolerant networks". In IEEE Communications Society Conference on Sensor, Mesh and Ad Hoc Communications and Networks (SECON), 2008.

[14] Indgren and K. S. Phanse, "Evaluation of queuing policies and forwarding strategies for routing in intermittently connected networks,"in Proc. of IEEE COMSWARE, Jan. 2006 .

[15] Sulma Rashid,Qaisar Ayub,"Effective buffer management policy DLA for DTN routing Protocals under congestion", International Journal of Computer and Network Security,Vol 2,NO 9,Sep 2010. pp .118-121

[16] Yun Li, Ling Zhao ,Zhanjun Liu,Qilie Liu.” N-Drop Congestion Control strategy under Epidemic Routing in DTN." Research center for wireless information networks, Chongqing University of posts \& Telecommunications, Chongqing 400065, china, pp. 457-460, 2009.

[17] Qaisar Ayub, Sulma Rashid," T-Drop: An optimal buffer management policy to improve QOS in DTN routing protocols", Journal of Computing, Vol 2, ISSUE 10,

[18] Rashid, S., et al., E-DROP: An Effective Drop Buffer Management Policy for DTN Routing Protocols. International Journal, 2011. 13(7): p. 8-13.

[19] Saad, M.I.M. and Z.A. Zukarnain, Performance Analysis of Random-Based Mobility Models in MANET Routing Protocol. European Journal of Scientific Research, 2009. 32(4): p. 444-454.

[20] Abdulla, M. and R. Simon. The impact of the mobility model on delay tolerant networking performance analysis. 2007: IEEE.

[21] T. Spyropoulos, K. Psounis, and C. Raghavendra A, C. S. "Single-copy routing in intermittently connected mobile networks," IEEE/ACM Transactions on Networking (TON), vol. 16 , pp. 63-76, Feb. 2008.

[22] Giudici, F., E. Pagani, and G.P. Rossi, Impact of Mobility on Epidemic Broadcast in DTNs. Wireless and Mobile Networking, 2008: p. 421-434. 